\title{
Study on the Fixture Analysis and Configuration Method for Computer-aided Modular Fixture Design
}

\author{
Y.Q. Zhou, B.Z. Li, Q.X. Wang \\ School of mechanical engineering, Donghua University \\ Shanghai China
}

\author{
Y. Zhang \\ Chongqing University \\ Chongqing, China
}

\begin{abstract}
Modular fixture design is a complex process. Computer-aided modular fixture design can help to accumulate the experience and technology of fixture designer. In this paper, the optimal configuration method of hole modular fixture is studied in computer-aided modular fixture design. Firstly, the characteristics of the modular fixture is analyzed, with the detail information needed during the fixture design process given, including the structure information, function information and assembly feature information of the element. Fixture information description model is built to support the database design of the modular fixture and the automatical generation of the modular fixture. An optimized configuration algorithm is studied to generate the modular fixture from the fixture elements to meet the requirements. Finally, an illustration is provided to testify the feasibility the algorithm.
\end{abstract}

Keywords-modular fixture; optimal configuration; fixture analysis

\section{INTRODUCTION}

During manufacturing, the workpiece geometric accuracy mainly depends on the relative position of the workpiece and machine tools, which should be guaranteed by fixture. So, fixture design is one of the important contents of the manufacturing process [1-2]. For modular fixture design, part of the elements can be selected to assemble into a variety of fixture used on the machine, according to the different machining process requirements. After using, the modular fixture can be disassembled into elements and components for new workpiece. The design and assembling of modular fixture is a complex process. The result greatly depends on the workers with rich experience in the production and skilled technical, which limit the widely use of modular fixture[3]. The study and research on computer-aided modular fixture design system can help to resolve this problem.

More and more researchers have studied on the computeraided modular fixture design. They are mainly focusing on fixture layout design, selection of locating and clamping point, the stiffness analysis and automatic generation algorithm of fixture structure design etc. [4]. Paper [5] gave a fixture information coding system in computer aided fixture design, to describe the fixture feature of workpiece. Paper [6] study on the automatic generation of fixture structure design, with an assembling relationship fig. of modular fixture element given. Paper [7] present an automatic generation algorithm of fixture based on case reasoning.
Database is an important part of computer-aided fixture design system. While generating the fixture automatically, algorithm is used to search the required fixture elements from the database to assemble into the modular fixture of the part. In this paper, an optimized configuration algorithm is studied. The structure information and function information of fixture elements are analyzed in detail, with other information needed during fixture design analyzed. An algorithm is present to generate the modular fixture from the fixture elements to meet the requirement.

\section{FEATURE ANALYSIS OF MODULAR FIXTURE}

When designing modular fixture, fixture layout scheme, locating and clamping point should be determined firstly. Then the modular fixture should be designed at the determined locating point to satisfy the requirements, which is the content of fixture optimized configuration. The fixture elements number is huge. The modular fixture designed for a workpiece is various. So, the optimized configuration algorithm should be studied to give the optimal rules of designing modular fixture, with structure and assemble feature of fixture elements analyzed and workers design experience studied. The optimal design method of modular fixture is studied as followed, taking the Hole Series modular fixture as an example. The structure of modular fixture is studied to analyze the recorded information.

\section{A. Structure Feature Analysis of Fixture}

Usually, modular fixture consists of several components, with locating and clamping function. Each component consists of one or more elements according to certain assembly relationship together. Each fixture element comprises several functional surface, namely, positioning, clamping or supporting surface etc. There are assembly features on the functional surface, such as pin holes, sink holes, and screw holes.

The fixture can be regarded as a set of units. Each unit can be regarded as a set of components. $F$ is used to represent the fixture, $U i$ denotes the $i$ th unit in fixture $F$. Cij denotes the component No. of unit $U i$. Then $F$ can be represented as:

$$
\begin{aligned}
& F=\left\{U_{i} \mid i=1,2, \ldots n_{u}\right\} \\
& U_{i}=\left\{c_{i j} j=1,2, \ldots n_{o i}\right\}
\end{aligned}
$$

So, 


$$
\left.F=\left\{\left\{c_{i j}\right]=1,2, \ldots, n_{c i}\right] \mid i=1,2, \ldots n_{u}\right\}
$$

Where, $n u$ denotes the number of fixture unit, $n c i$ denotes the component number of unit $U i$.

During fixture design progress, fixture components are to be selected from the database to assemble into needed modular fixture. Therefore the analysis of fixture element information is the most basic. The structure of fixture element is analyzed in detail to get the necessary information during fixture automatic design.

\section{B. Structure and Information Analysis of Fixture Element}

Hole series modular fixture elements can be divided into 5 categories: basic element, supporting element, positioning element, clamping element, subassembly and accessories. Each category of element can be divided into many kinds, according to the shape and the structure. In order to manage the element data, the same data format is analyzed to describe the element.

Each element consists of various functional surface with assembly features on it. Fixture element can be an element supporting other element or workpiece, or be an element supported by other element. Functional surfaces include supporting surface and supported surface. The assemble feature on the surface includes positioning holes, sink holes, screw holes, fastening sinking groove, fastening key slot, fastening $U$ sinking groove and fastening $U$ key slot. Positioning holes, sink holes, fastening sinking groove, fastening key slot, fastening $U$ sinking groove and fastening $U$ key slot on the supported surface can be used to locate and fasten the supported element on the supporting element. These features are defined as assembly features related to the supported surface. While positioning holes, screw holes, fastening key slot and fastening $U$ key slot on the supporting surface can be used to locate and fasten the supported element. These features are defined as assembly features related to the supporting surface. By analyzing the structure and information needed to be saved of the modular fixture elements, the structure and information figure is presented, as shown in figure 1 .

The fixture element information needed to be recorded includes: the basic information, geometrical information and assembly information. The basic information records the ID, name, type, functional type and assembly feature of the element mainly. The type records the category of element. The base plate includes horizontal base plate and vertical base plate. The positioning element includes bottom positioning element, side positioning element, bottom-side combined positioning element, inner hole positioning element and outer circle positioning element (V-block). The clamping element includes top-clamping element and side-clamping element. The supporting element includes clamping supporting element, bottom positioning supporting element and side positioning supporting element. The accessory includes nut, screw and spacer etc.

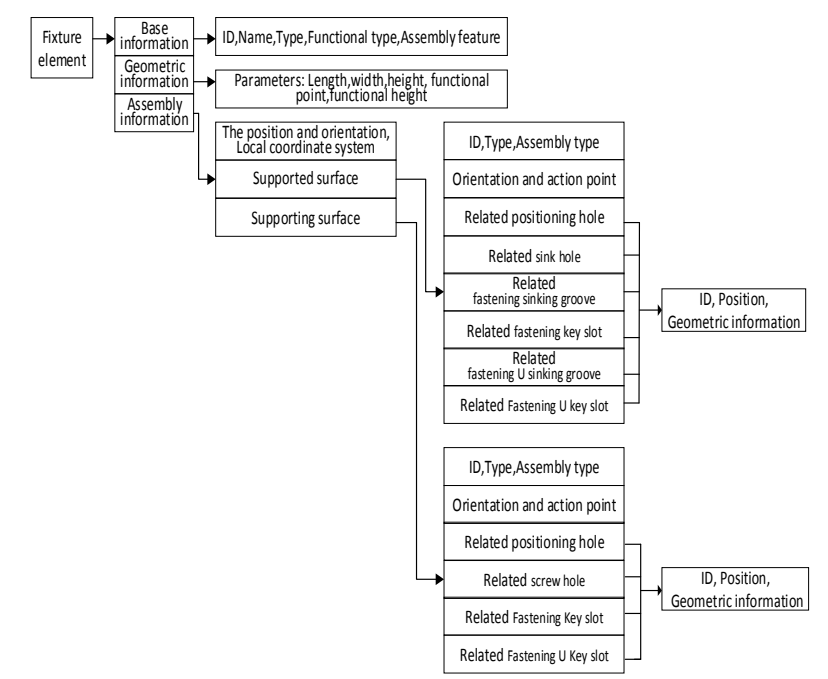

FIGURE I. STRUCTURE AND INFORMATION FIGURE OF FIXTURE ELEMENT.

Based on the function of fixture component, component includes vertical positioning component, horizontal positioning component, vertical-horizontal positioning component, vertical clamping component, horizontal clamping component, clamping supporting component, vertical supporting component and horizontal supporting component. The component is consist of elements. The function type of the element recorded is that which type of component it can be for. The same element may have different function type. It can compose a vertical positioning component with other elements, and it can also compose a vertical supporting component with other elements. UType is used to represent the type set of component. UFijk is used to represent the function type set of fixture element $c i j$. That is what type of component the element can be for. Then,

$$
U F_{i j}=\left\{U F_{i j k} \in U T y p e \mid k=n_{u f i j}\right\}
$$

Where $n_{u f i j}$ is the number of component type the element can form.

The geometric information of fixture element records mainly the geometric parameters, function point and function height of the element. Geometric parameters are different for different types of elements. For base plate, supporting plate, locating element, the length, width and height should be recorded. For V-block, the angle, the height of the intersection point to the end surface, the maximum and minimum outer diameter of element supported should be recorded. For the clamping element, length, width, height range, clamping travel range and assembly adjustable range should be recorded.

The assembly information of fixture element includes the location and orientation of the fixture element, local coordinate system information and the functional surface with its assembly features included. The surface ID, type, assembly type and orientation, related hole ID, location and geometric information are recorded. 


\section{Assembly Feature Analysis of Fixture}

The base assembly features information of the fixture element mainly records those elements it can be assembled together. Assembly relationship between fixture element is not any freedom, and to be subject to certain constraints. Some elements can only directly contact the workpiece for positioning action, and some elements may support other elements, and some elements can only directly be placed on the base plate, cannot be supported by other components. Taking hole modular fixture as example, the fixture element assembly features is analyzed. The assembly features of elements can be divided into the following categories.

The first type: The element can only be assembled on the base plate as supporting element. It cannot be supported by other elements, such as four-surface supporting element, clamping supporting element. These two elements can be assembled on the same type elements as self-supporting fixture elements.

The second type: The element can only directly contact with the workpiece, for positioning action, assembled at the top of the component. The element cannot support other elements. Trimming cylindrical bearing, step-cylindrical bearing, round pad, adjustable stop pin, V-block, the top clamping component and side clamping component are this type of element. Round pads can support similar pad to adjust the height of assemble component

The third type: The element can either be supported, or support other elements, i.e., either as a padded support, or as a positioning element, such as square chamfered step support, the bar step support, adjustable positioning bar plate. A bar support can be mounted on the top surface of the square step support, which can be placed on the top surface of the foursurface support. The step of the support can position with vertical and horizontal positioning, which is recorded in the functional type information of the element. For example, for bar step support, its functional type set is \{vertical positioning element, vertical and horizontal integrated positioning element\}.

By analyzing, assembly feature set UAssem is defined as UAssem $=\{$ whether may it be supported, whether may it support, whether may it locate or clamp directly, whether may it be self-supporting \}. For four-surface support and clamp support, the assembly feature set $U A$ ssem $=\{0,1,0,1\}$. 0 means no, 1 means yes. That is for four-surface support and clamp support, they may not be supported, may support, may not locate or clamp directly and may be self-supporting. For trimming cylindrical bearing, step-cylindrical bearing, adjustable stop pin, V-block, the top clamping component and side clamping component, the assembly feature set UAssem $=\{1,0,1,0\}$. For round pad, the assembly feature set UAssem $=\{1,0,1,1\}$. For square chamfered step support, the bar step support, adjustable positioning bar plate, the assembly feature set $U A$ ssem $=\{1,1,1,0\}$. After recording the assembly feature information of fixture element, when selecting elements to build component, the elements that can be assembled together will be selected.

\section{OPTIMAL CONFIGURATION ALGORITHM OF MODULAR FIXTURE}

Based on the analysis above, the element information of the modular fixture is recorded, including the base information, functional information and assembly feature information etc. Also the assembly relationship information between functional elements is recorded. When designing modular fixture, search related elements to form the required component according to these recorded information. An optimal configuration algorithm of modular fixture is studied, as the process is given as followed.

Step 1: Input the type $j$ and action height $H$ of the required fixture component.

Step 2: Based on the function type $\mathrm{j}$ of the component and the function information of the element, select the elements with the second or the third type of assembly feature. That is to select the elements which may locate or clamp directly, and the function height is less than $H . C_{n}=\left[C_{i} \in C_{j}\left[h_{i} \leq H\right]\right.$.

Step 3: Select the element in $\mathrm{Cn}$ as supported element sequentially, then search its supporting elements based on the assembly relationship information of the element with the accumulated functional height less than or equal to $H$. Or search the element with the first or third type of assembly feature based on the element's function information while the accumulated functional height less than or equal to $H$. Record all the possible element set.

Step 4: If the accumulated height is less than $H$, then select the searched supporting element as supported element sequentially, continue search the supporting element with accumulated height less than or equal to $\mathrm{H}$ based on the assembly relationship information and function information of the elements, according to the method in step 3. Add the new element into the element set searched before. Report this process until the accumulated height equal to $H$.

Step 5: Form the required component by all the elements with the accumulated function height equal to $\mathrm{H}$. Record the element combination sequentially, forming component assembly information. Using the three-dimensional CAD software to designate the assembly relationships between the various elements of the components and record

Record and store all the information to database for searching when using the optimal configuration algorithm to form the modular element in the computer-aided modular fixture design system.

\section{ModulaR FIXTURE DESIGN CASE}

As shown in figure 2, on the left of the workpiece, it needs horizontal and vertical locating on the bottom and side surface. The distance from the left bottom surface to the base plate is $155 \mathrm{~mm}$ (as shown in fig.2(a)). According to the horizontal and vertical locating function type and height requirements, use the optimal configuration algorithm to search the fixture element database to form the component 020-052-343 needed (As shown in fig.2(b)). The component is composed of the forsurface support 310-020-3-1, Square chamfered step support 310-052-1-1 and bar step support 310-343-7-1. The locating 
figure is shown in fig.3(c). The component can meet the horizontal and vertical locating requirements.

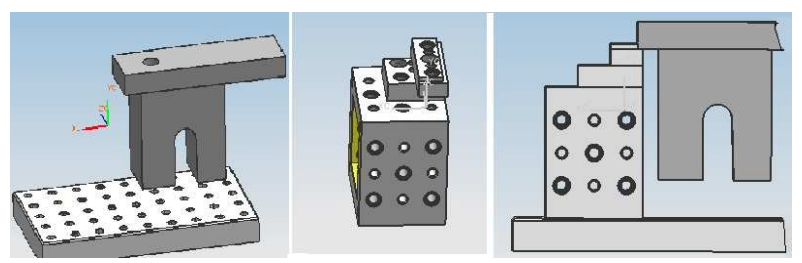

FIGURE II. COMPONENT DESIGN CASE.

\section{CONCLUSION}

In this paper, the structure of the hole series modular fixture element is analyzed in detail. Each element is decomposed into various functional surfaces with assembly features on it. The necessary information of the fixture element to be recorded includes base information, geometry information and assembly information. The element will be selected automatically to form the required component based on the information recorded. The optimal configuration algorithm of modular fixture is proposed. The fixture information describing model built and the optimal configuration algorithm of modular fixture given can provide a basis for the database built and automatic fixture component generation in the computer-aided fixture design system.

\section{REFERENCE}

[1] Y. Rong, Y. Zhu, L. Bi, computer-aided fixture design, Machinery Industry Press, 2002

[2] B.S. Thompson, M.V. Gandhi, Commentary on Flexible Fixturing, Applied Mechanics Review, 39(9): p.1365 1369, 1986.

[3] H. Zhao, Y. Zeng, Computer simulation of modular fixture assembly and Information Management, Aerospace Manufacturing Technology, 4(2), p.37-41,2004.

[4] J. Cecil, Computer-Aided Fixture Design- A Review and Future Trends, the International Journal of Advanced Manufacturing Technology, 18,p.790-793, 2001.

[5] Y. Rong, J. Zhu, and S. Li, Fixturing Feature Analysis for ComputerAided Fixture Design, Manufacturing Science and Engineering, ASME WAM, New Orleans, LA, Nov.28-Dec.2, PED-Vol. 64,p. 267-271, 1993.

[6] Y. Rong, Y. Bai, Automated Generation of Fixture Configuration Design, Journal of Manufacturing Science and Engineering., 119,p.208-219,1997.

[7] H. Wang, Y. Rong, Case Based Reasoning Method for Computer Aided Welding Fixture Design, Computer-Aided Design8,40,p.1121-113,2002. 